\title{
Re:Cycle - A Generative Ambient Video Engine
}

\author{
Jim Bizzocchi \\ Assistant Professor, School of Interactive Arts and Technology \\ Simon Fraser University \\ Canada \\ jimbiz@sfu.ca
}

\begin{abstract}
Re:Cycle is a visual poem, improvised in real-time by a system of deceptively simple rules relating to luminance and chrominance values in a set of moving image shots. Richly textured natural images of snow, mountains, rivers, and sky slowly unfold in a never-ending sequence that constantly changes. The resulting complex ambient video repudiates the standard cinematic conventions of linear narrative, and draws the viewer into an active creation of meaning for the work.
\end{abstract}

\section{Ambient Video}

Ambient Video is video intended to play on the walls in the backgrounds of our lives. In the spirit of Brian Eno's "ambient music", Ambient Video must be "as easy to ignore as it is to notice". [1] Eno's dictum can be expanded to three interrelated criteria that an ambient video art piece must meet. First, it must not require your attention at any time. Second, it must reward your attention with visual interest whenever you do look at it. Finally, because ambient pieces are designed to play repeatedly in our homes, offices and public spaces, they must continue to provide visual pleasure over repeated viewings. The ubiquitous screens in our domestic, corporate and social environments provide rich ground in which ambient imagery can thrive.

\section{The Creative Challenge}

The viewer's orientation to an ambient video work can be seen as a subtle dance, or to use Cubitt's term, a "dialogue" between the "system-cinema" and the autonomous viewer. [2]. The goal of Re:Cycle is to create an ambient work that will run indefinitely, and do so without repeating either shot sequences or specific transitional moments. This increase in playability does come with a price - a loss in aesthetic control over the details of sequencing and transition. One can see this as a tension built into the system. Other artists working in generative visual art have faced a similar set of balances and trade-offs. Lev Manovich's Soft Cinema [3] aims to combine the demands of narrative coherence with a recombinant database aesthetic. 77 Million Paintings by Brian Eno [4] is an extension of his own earlier linear ambient video work into a generative form. Re:Cycle is situated somewhere between 
these works. Like Soft Cinema it relies on recorded video and representational visuals, not on pure graphic material. At the same time, like 77 Million Paintings, Re:Cycle rejects narrative and is therefore more free to rely on completely random recombination to support re-playability.

The development of Re:Cycle has been a dialectical process - the balance between aesthetic control and re-playability has been revisited at every stage. One can frame each significant creative decision an attempt to maximize success across both ends of a continuum:

\section{re-playability <=========> aesthetic control}

Re-playability could be increased with a larger database of shots. However, an indiscriminate increase in numbers could undermine aesthetic impact. To avoid this, any additional shots selected would need to be of the same high visual quality as the original set. We limited our choice of transitions in order to protect aesthetic quality. There are a host of video transitional devices: the hard cut, the dissolve, innumerable shape-based wipes, and the more complex transitions such as luminance keyed transitions and chrominance keyed transitions. ${ }^{1}$ We decided to forego most of these possible transitions, and restrict ourselves to luminance and chrominance keys precisely because they were more visually complex and therefore more interesting to watch. They have the associated advantage that as the viewer watches them unfold, the flow and the details of the transition are less predictable than dissolves or simple shape-based wipes.

However, the luminance and chrominance keys were not the perfect aesthetic solution for all shots. We used two tactics to increase their aesthetic utility. Shots with very strong regional contrasts simply did not transition well from many of the other shots in our shot database. We removed some of those shots, sacrificing some replayability because of decreased shot numbers, but increasing our aesthetic impact through the avoidance of a number of poor transitions. Our second tactic was to increase the "feathering" of the edges of the wipes. "Feathering" refers to the softness of the edge of the transition as it proceeded. A sharp transition often produced a feeling of random video noise and visual "busy-ness". A softer transition was slightly less dramatic in some cases, but over the range of random shot transitions tended to be less noisy and more aesthetically pleasing.

\section{A Generative Solution}

A generative ambient video piece can use simple computational capabilities to continuously vary the sequencing and combinations of the selected ambient shots and visual transitions. Computational variability can further extend the ambient work's ability to use visual pleasure to slow perceived time and privilege reflection and contemplation.

Re:Cycle is a closed generative system, relying on two databases for its operation. The first is a database of ambient video shots, the second is a database of transitions.

\footnotetext{
${ }^{1}$ The terminology "wipes", "keys", and "keyed transitions" derives from the earlier language of cinema and analog video production and post-production.
} 
The twenty video clips in the shots database are all visually strong, at least sixty seconds long, and shot in the same general region (the Canadian Rockies). Many contain cyclical and visually interesting motion of clouds or water. The time frame in several of these shots has been manipulated in order to give the motion even more visual interest.

There are four transitions in the transitions database. The luminance transition uses the brightness values within the shot to drive the change from one shot to its successor. The incoming shot will appear first in the brightest sections of the current shot, then in the mid-range brightness areas, and finally in the darkest areas. When the transition is complete, the second shot has replaced the first completely. The other three transitions work in a similar fashion, except they are based on chrominance values (red, blue and green), not brightness. Each of these starts the transition in the areas of the shot with the highest chroma value in the selected color, and continues the transition down through the range of chroma saturation until the transition from one shot to the next is complete.

This engine has the capacity to present an ambient video art work that can run indefinitely, and still provide interesting visuals and transitions. The resultant doubly randomized video stream will generally not repeat particular shot sequencing with any frequency, and will generally provide a different transition for each change. The recombinant aesthetic will play out both temporally and spatially. The random sequencing will provide temporal recombination, while the interplay of random shot and transition selection will drive each shot change with a fresh spatial recombination.

\section{Future Work}

In addition to collecting a larger number of strong shots for our shot database, we will explore two other strategies: increasing the variety of shot transitions, and incorporating the use of metadata to increase aesthetic impact.

First, one can run any of our transitions in two directions. By incorporating both directions for each of our four transitions, we will increase our effective number of transitions to eight. Secondly, one could use either the current shot, or in the incoming shot as the basis for the transition. The number of possible transitions doubles again, resulting in a database with sixteen possible variations. We increase the re-playability of the piece, with no loss in aesthetic control.

We plan to associate metadata with individual shots, and use the metadata to guide sequencing and transition. This will increase aesthetic control, but it will also require more careful planning, and have a cost in variability and re-playability. The metadata will be used to encourage certain sequences of shots and transitions, and to block others. For example, we have observed that for some shots, certain transitions tend to work well. We've seen that shots that are heavy in red tones throughout respond unpredictably to transitions based on green chrominance values. We could use metadata to tag these shots, and block green chroma transitions. In doing so, we will reduce variability but ensure a higher level of aesthetic quality.

Metadata can also be used to give a level of editorial flow to the sequence. The categorization of shots (e.g., rocks, streams or clouds) can be used to favor sequences that are informally grouped to support semantic or aesthetic connections. Another 
example might be temporally based sequences that generally progressed from morning to mid-day to dusk, or from spring to summer and winter. All of these metadata applications are appealing because they are an opportunity to increase the coherence of the image flow; however there will be a commensurate loss in overall variability and re-playability. A more varied database of shots will mitigate this problem and allow us to further maximize Re:Cycle's aesthetic impact while maintaining our level of variability and re-playability.

Acknowledgements. Support for this project was received from the School of Interactive Arts and Technology and Simon Fraser University, by the Social Science and Humanities Research Council of Canada, and by the Banff New Media Institute. Re:Cycle imagery reflects the talent of Director of Photography, Glen Crawford. I also want to recognize the contribution to the work of my students, Brian Quan, Majid Bagheri, and Wakiko Suzuki.

\section{References}

1. Eno, B.: Music for Airports. Album liner notes in CD. PVC 7908, AMB 001 (1981)

2. Cubitt, S.: The Cinema Effect. MIT Press, Cambridge (2004)

3. Manovich, L.: Soft Cinema: Navigating the Database. DVD-video. MIT Press, Cambridge (2005)

4. Eno, B.: 77 Million Paintings by Brian Eno. Software program on DVD. All Saints Studio (2006) 